\title{
Success Rates for Functional MR Imaging in Children
}

\author{
A. Rajagopal, A. Byars, M. Schapiro, G.R. Lee, and S.K. Holland
}

\section{ABSTRACT}

BACKGROUND AND PURPOSE: Functional MR imaging is widely used for research in functional brain development in healthy children. However, obtaining high-quality brain imaging data from pediatric research participants requires cooperation that is challenging for young children. In this study, we examined success rates for fMRI in typically developing children in both longitudinal and cross-sectional research study designs to inform the recruitment needs of future pediatric brain imaging studies.

MATERIALS AND METHODS: In the cross-sectional study, 459 healthy children (5-18 years of age, 215 girls) were recruited. A subset of 30 healthy children 5-7 years of age from the cross-sectional cohort were selected and scanned for 10 consecutive years in the longitudinal arm of the study. Following anatomic scans, each participant attempted 4 functional MR imaging tasks. Success rate was defined as the proportion of fMRI tasks completed. Differences in success rates across sexes and in cross-sectional-versus-longitudinal cohorts were evaluated by using the Fischer exact test.

RESULTS: In the cross-sectional study, $74 \%$ of the children completed all tasks. Success rates for individual tasks ranged from $34 \%$ to $67 \%$ for children $5-7$ years of age and $76 \%-100 \%$ for those $8-18$ years of age. In the longitudinal study, $89 \%$ of children completed all tasks in all 10 years. We established significance $(P<.0001)$ between the cross-sectional and longitudinal cohorts for both $0 \%$ and $100 \%$ task completion rates. There was no significance between sexes.

CONCLUSIONS: When designing pediatric $\mathrm{fMRI}$ studies in children, the sample sizes indicated by power analysis should be scaled up according to age (ie, $33 \%$ for ages $8-18$ years, $50 \%$ for ages $5-7$ years).

ABBREVIATIONS: $\mathrm{HUSH}=$ hemodynamic unrelated to scanner hardware; $\mathrm{SR}=$ success rate

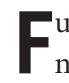
unctional MR imaging is a commonly used noninvasive technique for tracking the changes in blood oxygenation levels that accompany neuronal activity. ${ }^{1}$ Clinically, fMRI has been used extensively to identify or locate important brain regions before performing surgery on tumors or removing epileptic foci. ${ }^{2,3}$ It is also a widely used research tool for the investigation of brain activity during a wide array of primary somatosensory, language, and other higher level cognitive tasks. Most functional MR images

Received February 26, 2014; accepted after revision May 6

From the Pediatric Neuroimaging Research Consortium (A.R., G.R.L., S.K.H.), Department of Radiology, and Department of Neurology (A.B., M.S.), Cincinnati Children's Hospital Medical Center, Cincinnati, Ohio; and Departments of Radiology (S.K.H.), Pediatrics (S.K.H.), Otolaryngology (S.K.H.), Physics (S.K.H.), Neuroscience (S.K.H.), and Biomedical Engineering (S.K.H.), University of Cincinnati, Cincinnati, Ohio.

This work was funded by a grant from the National Institutes of Health (R01-HD38578).

Please address correspondence to Akila Rajagopal, MD, Pediatric Neuroimaging Research Consortium, Cincinnati Children's Hospital Medical Center, MLC 5033, 3333 Burnet Ave, Cincinnati, OH 45219; e-mail: akila.rajagopal@cchmc.org

- Indicates open access to non-subscribers at www.ajnr.org

http://dx.doi.org/10.3174/ajnr.A4062 require the subject to be awake and attentive to respond to the experimental paradigms being presented. The main challenges in successfully obtaining MR imaging data are restlessness, claustrophobia, or other anxieties on the part of participants.

The success rate (SR) of scanning children in fMRI paradigms has been examined in 2 studies. ${ }^{4,5}$ The study by Byars et $\mathrm{al}^{4}$ examined the success rate in 209 children between 5 and 18 years of age completing $4 \mathrm{fMRI}$ tasks and 1 anatomic reference scan. In that study, most of the children in the age range of 9 through 18 years completed all 4 functional tasks and the anatomic task. However, the failure rate was higher in younger children 5-7 years of age. The current sample of 459 children included the original group of $200+$ subjects from Byars et al in 2002, plus an additional 259 subjects in the cross-sectional cohort.

In another recent study, children in the age range of $10-18$ years had a significantly greater scan success rate than those 4-6 years of age. Yerys et $\mathrm{al}^{5}$ determined that groups with clinical conditions like attention deficit/hyperactivity disorder, autism spectrum disorder, and epilepsy had lower success rates than nonclinical control groups. 
In the present report, success and failure rates were examined in both cross-sectional and longitudinal cohorts. The longitudinal cohort of $n=30$ subjects was a subset of the cross-sectional cohort. This group of children was tested annually for 10 years. In the current report, we also include analysis and discussion regarding the reasons that children fail to complete fMRI scans for research. This information may be helpful to the researcher to avoid loss of subjects and data in pediatric neuroimaging studies and to provide a better understanding of the recruitment needs in future studies.

\section{MATERIALS AND METHODS Subjects}

Four hundred fifty-nine healthy children (215 girls) within the age range of 5-18 years were recruited for the cross-sectional fMRI study. The 3 criteria for recruitment were that children be healthy (determined by a questionnaire filled out by the parent), be native English speakers, and have normal findings on neurologic examinations administered by a board-certified pediatric neurologist. Children with any neurologic comorbidity were excluded from the study. From these 459 children, a subset of 30 children (15 girls) within the initial age range of 5-7 years was selected for the longitudinal fMRI study. For this study, the longitudinal study required annual fMRI scanning and testing for 10 consecutive years. The first visit of each longitudinal subject was included in the cross-sectional data analysis described below. Subsequent visits by longitudinal subjects were not included in the cross-sectional analysis. All visits by longitudinal participants are analyzed in the longitudinal trajectories.

\section{MR Imaging}

MR imaging was performed on a 3T Biospec MR imaging scanner (Bruker BioSpin MRI, Ettlingen, Germany). The entire scanning session lasted for approximately 45 minutes and included a localizer scan, anatomic scan, multiecho reference scan, and 4 functional MR imaging tasks. The T1-weighted 3D MPRAGE-type anatomic image was acquired in 9 minutes with $1 \times 1.5 \times 1.5 \mathrm{~mm}$ resolution. A T2*-weighted gradient-echo EPI sequence was used to acquire the functional scans. The imaging parameters used for these scans were as follows: $\mathrm{TR} / \mathrm{TE}=3000 / 38 \mathrm{~ms}$, bandwidth $=$ $125 \mathrm{kHz}$, matrix $=64 \times 64, \mathrm{FOV}=25.6 \times 25.6 \mathrm{~cm}$, section thickness $=5 \mathrm{~mm}$. In 5 minutes 30 seconds, 24-32 transverse sections were acquired.

\section{fMRI Paradigms}

Four functional tasks examined early and later developing sentential and syntactic language skills: verb-generation, syntactic prosody, picture-matching, and story-processing tasks. ${ }^{6}$

The verb-generation task ${ }^{7}$ involved auditory presentation of a series of concrete nouns every 5 seconds, to which the child responded by covertly generating as many verbs as possible associated with the noun during the remainder of the 5-second interval. A bilateral finger-tapping task served as the control task, preventing the child from continuing to generate verbs during the control period and providing a reference area of activation within the motor strip as a means of validating behavioral compliance.

The story-processing task ${ }^{8}$ involved an auditory presentation of 5 simple stories, each composed of 10 sentences. The child was instructed to listen to the stories in preparation for answering questions about them after the scans. Random auditory pure tones of various frequencies from 150 to $1000 \mathrm{~Hz}$ were presented at unequal intervals of $1-3$ seconds during the control task, which was interleaved by the story task.

In the syntactic prosody task, ${ }^{9}$ the child had an auditory presentation of a target sentence (selected from the stories in the story-processing task) of a set of sentences that were low-passfiltered ( $400 \mathrm{~Hz}$ cutoff) so that words were not recognizable, but syntactic prosody was preserved. During the control task, the child was asked to press a button each time a target tone was heard among the other randomly ordered tones of various frequencies.

The word-picture matching task ${ }^{10}$ involved simultaneous visual presentations of 2 simple line drawings of common objects. The name of one of the objects was presented via the headphones simultaneously. The child was required to push the button indicating whether the picture on the right or the left matched the word that was heard. The control task was a visual presentation of paired images of unnamable designs.

The prosody and story-processing tasks assess syntax, whereas word-picture matching and verb generation assess semantics. ${ }^{6}$ During the first 5 years in the longitudinal study, all children performed these 4 tasks. A nonsignificant rate of change in the lateralization index with age in the first 5 years of the longitudinal study indicated that picture-matching and prosody were early developmental tasks. Hence, in the last 5 years of the longitudinal study, these 2 tasks were replaced by modified versions of the story-processing ${ }^{11}$ and verb-generation tasks ${ }^{12}$ by using another method called hemodynamic unrelated to scanner hardware (HUSH). ${ }^{13}$ This method was designed to facilitate fMRI with auditory stimulation by presenting the stimulus when the scanner is completely silent, and the data are collected through the peak of the hemodynamic response. ${ }^{11}$ Other investigators have reported similar approaches. ${ }^{14}$

\section{Desensitization Methods}

To improve the success rate due to children being frightened, we used a systematic desensitization method to acclimate children to the MR imaging environment. This process begins with an experienced and child-friendly study coordinator and MR imaging technologist and continues with the following: 1) rewarding children with toys and small gifts for completing each training step, 2) acclimatizing them to the scanner by performing a trial run on the scanner, 3) explaining the procedures in child-friendly but detailed language and letting them hear the noise that the scanner makes, and 4) playing movies during nontask time in the scanner and frequently "checking in" with them by using the audio system while they are in the scanner. These systematic methods add approximately an hour of preparation time in the scanner area before the actual scan. Scanning procedures also last for almost an hour, with additional time between image sequences allocated to interaction with the participants by the study coordinator over the MR imaging-compatible audiovisual system to provide instructions and reassurance about performance and the time left until completion of the scan procedures. 


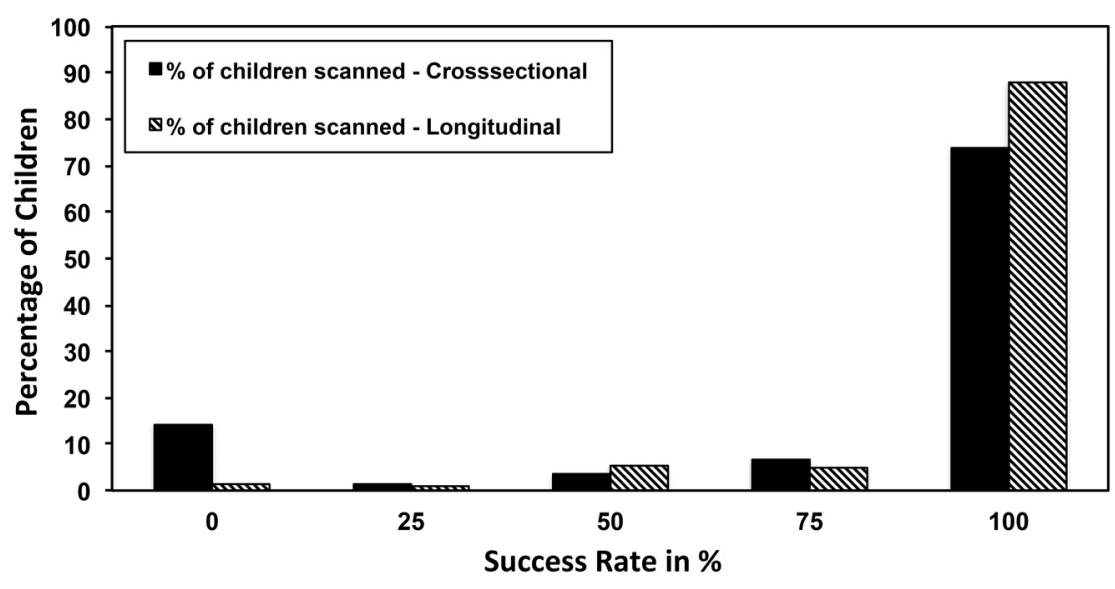

FIG 1. Histogram showing success rates of the percentage of children (y-axis) in the cross-sectional study $(n=459)$ and longitudinal study who were scanned for 10 years $(n=30)$, performing $4 \mathrm{fMRI}$ tasks, and their success rate ( $\mathrm{x}$-axis). $100 \%$ success rate $=4$ tasks completed; $75 \%, 50 \%, 25 \%$, and $0 \%$ correspond to $3,2,1$, or no task completed.

\section{Success Rate}

The success rate was defined as the ratio of the number of completed and usable fMRI datasets to the total number of fMRI tasks in the study protocol (ie, 4). FMRI data were considered usable if the subject completed the reference scan and anatomic scan with a minimum of motion, along with functional scans. ${ }^{15}$ The reference scan was considered important because it helps to perform the geometric correction during reconstruction of the images. ${ }^{16}$

\section{Reasons for Failure}

Reasons for children failing to complete MR imaging research protocols are important to understand for optimizing study designs for success. To quantitate reasons for failure, we define the 5 most common reasons for failure and examine the number of failures for each reason. Reasons for failure are defined in order of the largest to smallest number of children reporting this reason for ending participation in the scan session as follows.

1) Children being frightened due to claustrophobia or the noise generated by the scanner

2) No explanations or reasons provided by the subject or the technologists

3) Discomfort or unwillingness to lie still in the scanner

4) Technical errors made by the technologists or researcher. We lost a few data points due to trouble with the scanner or the stimulus-presentation software or video and audio equipment.

5) Motion inside the scanner. Children, especially in the age range of 5-7 years, found it hard to lie still in the scanner for a long time, with head or feet resulting in motion artifacts that caused the operators to stop the scan.

In the case of the longitudinal study, the primary reason for failure was children with braces or retainers, defined as reason 1 for the longitudinal cohort. Braces were exclusion criteria for the neuroimaging study of child language development, and children would not be recruited for the cross-sectional study if braces were discovered during the screening process. However, longitudinal participants who did not have braces when they first enrolled in the study (at age 5-7) were not excluded from continued participation in the longitudinal arm of the study but were not scanned in the years when they had braces. Among the longitudinal participants who continued in the imaging study, failure to complete scans in subsequent years was less common than in the cross-sectional group in general. However, longitudinal participants did fail to complete scans in subsequent years for the same reasons as cross-sectional participants with the 3 most common reasons for failure in the longitudinal study corresponding to items 2,3 , and 5 of the cross-sectional study listed above. The fourth most common reason for failure in the longitudinal study was families not being interested in continuing to participate in the study. Technical faults ( 4 above) were negligible in the longitudinal study because these "bugs" were eliminated during the first year or 2 of the study.

\section{Statistical Analysis}

We compared success/failure rates between boys and girls and also between the cross-sectional and longitudinal cohorts. To test the significance of the differences between the proportions in these groups, we used the Fisher exact test.

\section{RESULTS}

Success rate was computed separately for cross-sectional and longitudinal study data. The percentage of children who completed each fMRI task in the cross-sectional and longitudinal studies is plotted as a histogram in Fig 1. The percentages of children who did not complete any of the 4 functional scans, anatomic scan, or reference scan in the cross-sectional and longitudinal study are $14.4 \%(n=66)$ and $1.3 \%(n=3)$, respectively. In the following sections, the subject groups completing $0 \%$ through $100 \%$ of the scans will be referred to as SR(0) through SR(100). The number of subjects meeting SR(25), SR(50), SR(75), and SR(100) for the cross-sectional study are as follows: $1.3 \%(n=6), 3.7 \%(n=17)$, $6.8 \%(n=31)$, and $73.9 \%(n=339)$. The corresponding success rates for the longitudinal study are the following: $0.9 \%(n=2)$, $5.2 \%(n=12), 4.7 \%(n=11)$, and $87.9 \%(n=204)$, respectively.

\section{Cross-Sectional Cohort}

The number of children who completed each task was the following: picture-matching, 374 (81.5\%); verb-generation, 376 (81.9\%); syntactic prosody, 368 (80.2\%); and story-processing task, $370(80.6 \%)$. Table 1 shows the total number of children at each age and the percentage of children (girls and boys) in the cross-sectional study who completed each functional MR imaging task. Table 1 shows that the percentage of children completing each task was $>76 \%$ in the 8 - to 11 -year age group and $>91 \%$ in the 12 - to 18 -year age group. However, the success rates for the children in the 5- to 7-year age group ranged from $34 \%$ to $67 \%$. 
Table 1: The total number of girls and boys at every age ranging from 5 to 18 years participating in the cross-sectional study ${ }^{a}$

\begin{tabular}{|c|c|c|c|c|c|c|c|c|c|c|}
\hline \multirow[b]{3}{*}{ Age (yr) } & \multirow{2}{*}{\multicolumn{2}{|c|}{$\begin{array}{c}\text { Total No. of } \\
\text { Children }\end{array}$}} & \multicolumn{8}{|c|}{ Tasks Completed } \\
\hline & & & \multicolumn{2}{|c|}{ Picture (\%) } & \multicolumn{2}{|c|}{ Prosody (\%) } & \multicolumn{2}{|c|}{ Stories (\%) } & \multicolumn{2}{|c|}{ Verbs (\%) } \\
\hline & Girls & Boys & Girls & Boys & Girls & Boys & Girls & Boys & Girls & Boys \\
\hline 5 & 15 & 29 & 60 & 34.5 & 60 & 37.9 & 67 & 41.4 & 60 & 41.4 \\
\hline 6 & 22 & 23 & 63.6 & 47.8 & 54.5 & 39.1 & 59.1 & 47.8 & 54.5 & 43.5 \\
\hline 7 & 27 & 22 & 66.7 & 50 & 59.3 & 50 & 63 & 50 & 67 & 50 \\
\hline 8 & 15 & 25 & 80 & 88 & 87 & 84 & 80 & 84 & 80 & 92 \\
\hline 9 & 16 & 17 & 94 & 88 & 81.3 & 76.5 & 81.3 & 82.4 & 87.5 & 76.5 \\
\hline 10 & 12 & 17 & 100 & 88 & 100 & 94 & 92 & 82.4 & 100 & 82.4 \\
\hline 11 & 13 & 17 & 84.6 & 100 & 84.6 & 94.1 & 84.6 & 100 & 92.3 & 100 \\
\hline 12 & 14 & 22 & 100 & 100 & 100 & 100 & 100 & 95.5 & 100 & 100 \\
\hline 13 & 21 & 19 & 95.2 & 94.7 & 95.2 & 94.7 & 95.2 & 94.7 & 100 & 94.7 \\
\hline 14 & 14 & 12 & 92.8 & 100 & 92.8 & 100 & 92.8 & 100 & 92.8 & 100 \\
\hline 15 & 12 & 11 & 91.7 & 100 & 91.7 & 100 & 91.7 & 100 & 91.7 & 100 \\
\hline 16 & 11 & 10 & 100 & 90 & 100 & 100 & 100 & 100 & 100 & 100 \\
\hline 17 & 11 & 12 & 100 & 100 & 100 & 100 & 91 & 100 & 100 & 100 \\
\hline 18 & 12 & 8 & 100 & 100 & 100 & 100 & 100 & 100 & 100 & 100 \\
\hline
\end{tabular}

${ }^{a}$ Also tabulated is the number of girls and boys who completed the various functional MRI tasks.

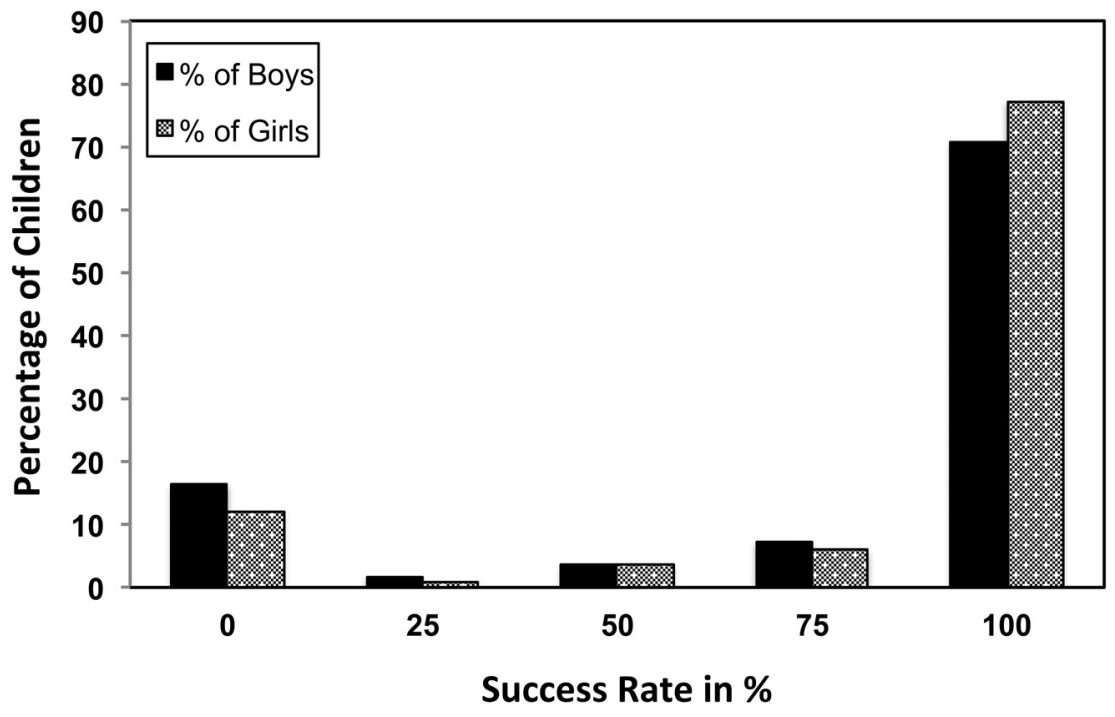

FIG 2. Histogram showing success rates of the percentage of girls $(n=215)$ and boys $(n=244)$ in the cross-sectional study along the $y$-axis and their success rates along the $x$-axis. $100 \%$ success rate $=4$ tasks completed; $75 \%, 50 \%, 25 \%$, and $0 \%$ correspond to $3,2,1$, or no task completed.

Figure 2 shows the success rates in the cross-sectional study based on sex. Twelve percent of girls tended to have SR $(0)$, while $16 \%$ of boys did not complete any of the tasks. In addition, $77 \%$ of the girls completed all the scans, while $71 \%$ of the boys completed all the scans.

\section{Longitudinal Cohort}

Figure 3 shows the number of children who completed the study annually in the longitudinal study grouped by the age of children in each year. Because the total number of subjects was limited to 30 , grouping by age was not performed for each task.

In Table 2, the percentage of children who completed tasks in the longitudinal study for each year for each individual task is tabulated. Table 2 also shows the number of children who were scanned for each year. There are a total of 6 tasks listed in Table 2; however, during the first 5 years of the study, the 4 tasks used were picture, prosody, stories, and verbs. During the last 5 years, the tasks used were stories, verbs, HUSH sto- ries, and HUSH verbs. On the basis of Table 2, it can be seen that the success rate was $>89 \%$ for the children who returned every year.

Tables 3 and 4 explain the reasons for failure in both cross-sectional and longitudinal studies. The criteria on the basis of which the subjects failed to either complete any task or 1 or 2 or 3 tasks are shown along with the percentage of children who failed for each reason.

\section{Statistical Significance}

The Fisher exact test on the success rates in the cross-sectional and longitudinal cohorts yielded a significant difference in the both $\operatorname{SR}(0)$ and $\mathrm{SR}(100)$ rates $(P<.0001)$. The differences at various other success rates, SR(25), SR(50), and SR(75), were not significant. We also tested for statistical significance between girls and boys in the cross-sectional cohort, but there was no significant difference.

\section{DISCUSSION}

The percentage of children in SR(100) was $89 \%$ for the longitudinal study compared with $74 \%$ in the cross-sectional study. The children in the longitudinal study had a better success rate because these children were, in most cases, comfortable with being scanned after the initial scanning and desensitization process. We expected 300 scans for the longitudinal study because we had 30 children recruited who were scanned for 10 years. However, a number of scans were omitted because the children had braces or retainers, and a few families were not interested in continuing to participate in the study or they moved.

From Table 1, it is clear that children 5-7 years of age had the lowest success rate in our study, ranging from $34.5 \%$ to $67 \%$. However, there was a drastic improvement in success rates in children 8 and 9 years of age, with success rates ranging from 
$76.5 \%$ to $94 \%$. Children in the 10 - to 18 -year age group showed success rate ranging from $82.4 \%$ to $100 \%$.

Girls tended to have lower failure rates and higher success rates than boys when considering SR(0) and SR (100) (Fig 2). However, both boys and girls had almost the same rate of success in completing 1, 2, and 3 tasks. Although girls had a higher success rate than boys, a Fisher exact test did not find this difference significant. We can conclude that it is reasonable to expect high rates of success in $\mathrm{fMRI}$ research studies in both boys and girls as young as 5 years of age.

We have previously shown that task order is not a factor in determining success rate. ${ }^{15}$ The main determinant of noncompliance with fMRI task demands by children is the degree of engagement of auditory, visual, and tactile senses, particularly in boys. Even though girls had a higher success rate than boys, the difference was not significant. The current results are consistent with our earlier findings in this regard. ${ }^{15}$

The success rate in the longitudinal study was calculated on the basis of the number of tasks completed by the children compared with the number of children who returned for scans. Children with braces or retainers who could not be scanned were not included in the analysis. On the basis of Table 2, the success rates for

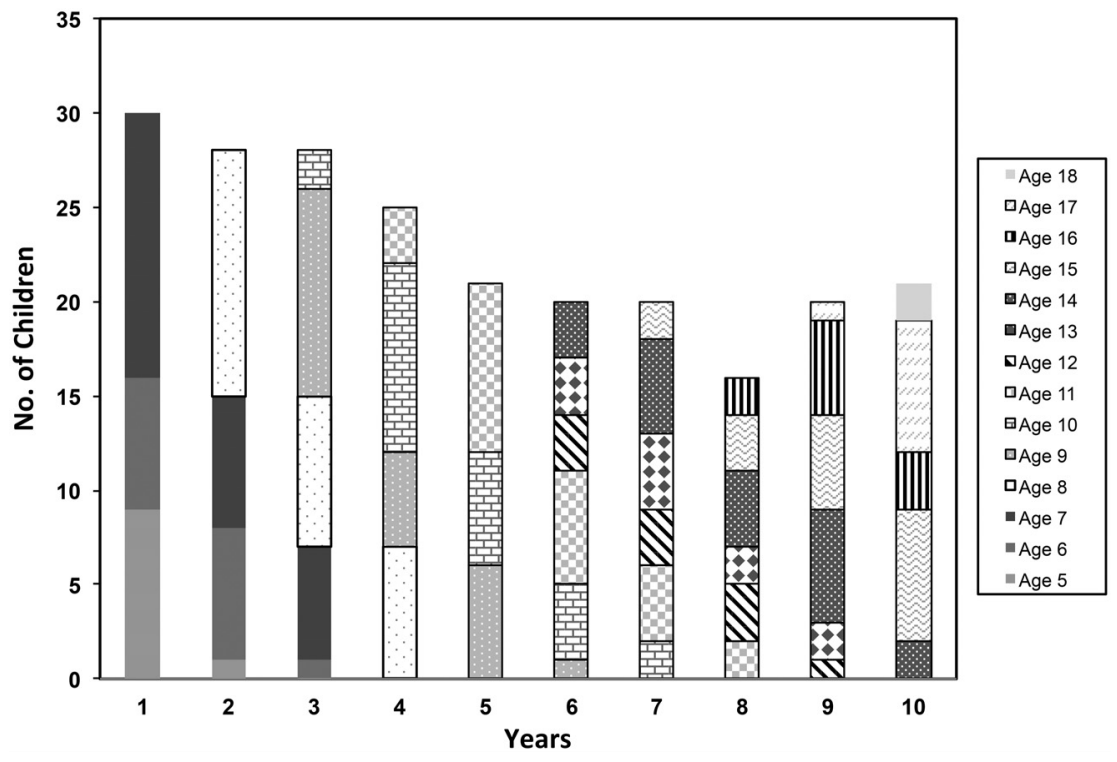

FIG 3. Histogram of the number of children (y-axis) scanned for the longitudinal study annually for 10 years ( $x$-axis). The histogram of the number of children is further sorted on the basis of the age of the children during their visit at each year (gray-scale pattern). years 1 and 4 were $>92 \%$, dropped a little in years 2 and 3 , but remained $>89 \%$. We had $100 \%$ success for the years $6,7,8$, and 10 and $>95 \%$ success rates for the fifth year. Note that success rates improved as children grew older and had more experience with the procedures.

In Tables 3 and 4, we can see that the primary reasons for failure in the longitudinal study were because the family lost interest or could not be contacted or children had braces in their teenage years. However, the most common reason for failure in the cross-sectional study was that children were afraid to be scanned, quit early, or had excessive motion. This suggests that once children are acclimated to being scanned, they are generally successful in subsequent longitudinal sessions. These Tables provide important information for those interested in conducting pediatric fMRI studies.

Desensitization methods, including a practice run on the MR imaging scanner, continual communication, feedback, and reassurance of children, have been found to be important to our success rates. Even though these procedures add time to the imaging session, they have helped us achieve a better success rate in the cross-sectional study and also to ensure that more children return for scans in the subsequent years of the longitudinal study. Annual holiday greeting cards, birthday cards, and a Facebook group for the longitudinal cohort have also improved our long-term retention as the children enter high school and college.

The results from the cross-sectional study show that $77.3 \%$ of the girls and $71 \%$ of the boys completed the scans successfully. For a cross-sectional study, recruitment should aim for four-thirds of the desired sample size in children ages $8-18$ years and 1.5 times in children ages 5-7 years to meet the power requirements of the study. Oversampling will allow a projected overall success rate of $75 \%$. In the longitudinal study, among the 30 children who were recruited, 3 quit the study either due to moving out of town or not wishing to participate further. On the basis of the numbers from Fig 2, it can be seen that during the fifth, sixth, and seventh years,

Table 2: Total number of children scanned longitudinally and the tasks completed in the scanner

\begin{tabular}{cccccccc}
\hline \multirow{2}{*}{$\begin{array}{c}\text { Study } \\
\text { Year }\end{array}$} & $\begin{array}{c}\text { Total Subjects } \\
\text { Scanned }\end{array}$ & Prosody (\%) & Picture (\%) & Verbs (\%) & Stories (\%) & Verbs HUSH (\%) & Stories HUSH (\%) \\
\hline & 30 & 90 & 86.7 & 86.7 & 93.3 & NA & NA \\
2 & 28 & 89.3 & 92.9 & 96.4 & 89.3 & NA & NA \\
3 & 28 & 89.3 & 96.4 & 92.9 & 92.9 & NA & NA \\
4 & 25 & 96 & 96 & 96 & 92 & NA & NA \\
5 & 21 & 95.2 & 100 & 100 & 95.2 & 100 & 100 \\
6 & 20 & NA & NA & 100 & 100 & 100 & 100 \\
7 & 20 & NA & NA & 100 & 100 & 100 & 100 \\
8 & 16 & NA & NA & 100 & 100 & 100 & 95 \\
9 & 20 & NA & NA & 100 & 100 & 100 & 100 \\
10 & 21 & NA & NA & 100 & 100 & & 100 \\
\hline
\end{tabular}

Note:-NA indicates not applicable. 
Table 3: Reasons for failure to complete fMRI tasks for the crosssectional cohort and the number of children who failed for each reason $^{\mathrm{a}}$

\begin{tabular}{lcccc}
\hline & \multicolumn{4}{c}{ Cross-Sectional } \\
\cline { 2 - 5 } \multicolumn{1}{c}{ Criteria } & SR(0) & SR(25) & SR(50) & SR(75) \\
\hline Frightened & 36 & 0 & 3 & 2 \\
No details & 16 & 0 & 0 & 1 \\
Patient quit & 8 & 4 & 10 & 17 \\
Technical fault & 0 & 0 & 1 & 7 \\
Motion & 3 & 2 & 2 & 5 \\
\hline
\end{tabular}

a The subject groups completing $0 \%$ through $75 \%$ of the scans are referred to as $\operatorname{SR}(0)$ through SR(75).

Table 4: Reasons for failure to complete fMRI tasks for the longitudinal cohort and the number of children who failed for each reason ${ }^{\mathrm{a}}$

\begin{tabular}{lcccc}
\hline & \multicolumn{4}{c}{ Longitudinal } \\
\cline { 2 - 5 } \multicolumn{1}{c}{ Criteria } & SR(0) & SR(25) & SR(50) & SR(75) \\
\hline Braces & 34 & 0 & 0 & 0 \\
No details & 20 & 0 & 0 & 0 \\
Not interested & 16 & 0 & 0 & 0 \\
Patient quit & 0 & 12 & 11 & 4 \\
Motion & 2 & 0 & 0 & 0 \\
\hline
\end{tabular}

a The subject groups completing $0 \%$ through $75 \%$ of the scans are referred to as $\operatorname{SR}(0)$ through SR(75).

fewer children completed the scans. This finding was mainly due to children in the age range from 11 to 18 having braces or retainers that were not MR imaging-compatible.

\section{Limitations}

This study is subject to a few limitations that may restrict its generalizability to other populations and centers. Most important, the study was conducted in healthy children who were recruited specifically for a neuroimaging research protocol. Consequently, these children come from highly motivated families who either have altruistic tendencies or an interest in their child's development. The sample may not be representative of healthy populations as a whole because of the increased motivation of the families to participate in a medical research project. Furthermore, this healthy population of children is most certainly not representative of children with various pathologies such as attention deficit/ hyperactivity disorder, autism, epilepsy, or other neurologic or psychiatric disorders. Consequently those designing studies with such populations may not be able to directly extrapolate from the failure rates reported in our study.

Another relevant limitation of the study is that it began in 2000 by using a prototype $3 \mathrm{~T}$ research MR imaging scanner. Technical advances in clinical MR imaging scanner designs operating at this field strength have resulted in much improved image quality and patient throughput. For example, improved laser alignment and prescan procedures have greatly accelerated the time needed to complete a neuroimaging research protocol. The data acquired for this study between 2000 and 2008 were obtained on a 3T Biospect 30/60 (Bruker) scanner with a $60-\mathrm{cm}$ bore and a quadrature head coil with a $210-\mathrm{mm}$ inner diameter. All of the cross-sectional participants in the study were scanned on this early $3 \mathrm{~T}$ prototype scanner with an accompanying rate of technical failure and other factors potentially impacting success rates. This configuration also necessitated using video goggles rather than a projection screen for visual presentation, which also may have impacted the failure rates.

In the final 2 years of the study, longitudinal participants were scanned on a more modern 3T Achieva (Philips Healthcare, Best, the Netherlands) MR imaging scanner. As with other modern clinical 3T MR imaging scanners, the system offers a wider bore, more open head coil design with an improved signal-to-noise ratio and image quality. Throughput is improved with better alignment tools and prescanning protocols, resulting in improved success rates for children. These factors are folded into the improved success rates we report for longitudinal subjects in later years and introduce confounding to our analysis.

Finally, with the wider installation of clinical 3T MR imaging scanners in research laboratories and clinical environments, more centers have begun to conduct neuroimaging studies of development in pediatric populations. Several centers, including ours, have developed desensitization protocols that involve pretraining of pediatric participants by introducing them to the noises that MR imaging scanners make for several days before the scanning session and training videos that introduce participants to the MR imaging scanner and laboratory environment before they arrive for their first session. ${ }^{17}$ These techniques have proved to work effectively to gain a higher level of cooperation from children. While the current study used some of these methods as we have reported previously, ${ }^{4,5}$ our newer methodology may result in better success rates than what we have reported here.

\section{CONCLUSIONS}

The success rates provided in this article are relevant for planning future cross-sectional or longitudinal functional imaging studies in normally developing healthy children. On the basis of the success rate analysis by age, when designing fMRI studies involving typical children, the sample size indicated by appropriate power analysis must be increased by $33 \%$ in ages older than 8 years and $50 \%$ in children 5-7 years of age. This percentage might have to be slightly higher for boys compared with girls because girls tend to comply with the behavioral demands of fMRI scans in the 5- to 18 -year age group. While success rates for longitudinal participants are higher than those for the cross-sectional population in general, powering a pediatric neuroimaging study design as outlined above will provide sufficient high-quality data to permit longitudinal data analysis for a study ranging up to 10 years in duration.

Disclosures: Akila Rajagopal—RELATED: Grant: National Institutes of Health R01HD38578.* Anna Byars—RELATED: Grant: National Institutes of Health.* Mark Schapiro-RELATED: Grant: National Institutes of Health, ${ }^{\star}$ Comments: I receive support from a National Institutes of Health contract. Gregory R. Lee-RELATED: Grant: National Institutes of Health: National Institute of Child Health and Human Development, Comments: primary source of funding for the study; Support for Travel to Meetings for the Study or Other Purposes: National Institutes of Health: National Institute of Child Health and Human Development. Scott K. Holland-RELATED: Grant: National Institutes of Health R01-HD38578*; Support for Travel to Meetings for the Study or Other Purposes: National Institutes of Health, ${ }^{*}$ Comments: presentations at Human Brain Mapping, American Society of Neuroradiology, and other meetings; UNRELATED: Grants/Grants Pending: National Institutes of Health R01HD38578, * Comments: no financial benefit to me personally or to my company. The grant was used exclusively to pay for the research study. *Money paid to the institution. 


\section{REFERENCES}

1. Ogawa S, Lee TM, Kay AR, et al. Brain magnetic resonance imaging with contrast dependent on blood oxygenation. Proc Natl Acad Sci US A 1990;87:9868-72

2. Hingwala D, Thomas B, Radhakrishnan A, et al. Correlation between anatomic landmarks and $\mathrm{PMRI}$ in detection of the sensorimotor cortex in patients with structural lesions. Acta Radiol 2014;55: 107-13

3. Zhang J, Mei S, Liu Q, et al. fMRI and DTI assessment of patients undergoing radical epilepsy surgery. Epilepsy Res 2013;104:253-63

4. Byars AW, Holland SK, Strawsburg RH, et al. Practical aspects of conducting large-scale functional magnetic resonance imaging studies in children. J Child Neurol 2002;17:885-90

5. Yerys BE, Jankowski KF, Shook D, et al. The fMRI success rate of children and adolescents: typical development, epilepsy, attention deficit/hyperactivity disorder, and autism spectrum disorders. Hum Brain Mapp 2009;30:3426-35

6. Holland SK, Vannest J, Mecoli M, et al. Functional MRI of language lateralization during development in children. Int J Audiol 2007; 46:533-51

7. Holland SK, Plante E, Weber Byars A, et al. Normal fMRI brain activation patterns in children performing a verb generation task. $\mathrm{Neu}$ roimage 2001;14:837-43

8. Karunanayaka PR, Holland SK, Schmithorst VJ, et al. Age-related connectivity changes in fMRI data from children listening to stories. Neuroimage 2007;34:349-60
9. Plante E, Holland SK, Schmithorst VJ. Prosodic processing by children: an fMRI study. Brain Lang 2006;97:332-42

10. Schmithorst VJ, Holland SK, Plante E. Object identification and lexical/semantic access in children: a functional magnetic resonance imaging study of word-picture matching. Hum Brain Mapp 2007;28:1060-74

11. Vannest JJ, Karunanayaka PR, Altaye M, et al. Comparison of fMRI data from passive listening and active-response story processing tasks in children. J Magn Reson Imaging 2009;29:971-76

12. Vannest J, Rasmussen J, Eaton KP, et al. FMRI activation in language areas correlates with verb generation performance in children. Neuropediatrics 2010;41:235-39

13. Schmithorst VJ, Holland SK. Event-related fMRI technique for auditory processing with hemodynamics unrelated to acoustic gradient noise. Magn Reson Med 2004;51:399-402

14. Birn RM, Bandettini PA, Cox RW, et al. Event-related fMRI of tasks involving brief motion. Hum Brain Mapp 1999;7:106-14

15. Yuan W, Altaye M, Ret J, et al. Quantification of head motion in children during various fMRI language tasks. Hum Brain Mapp 2009;30:1481-89

16. Schmithorst VJ, Dardzinski BJ, Holland SK. Simultaneous correction of ghost and geometrical distortion artifacts in using a multiecho reference scan. IEEE Trans Med Imaging 2001;20:535-39

17. Vannest J. Behavioral methods for successful non-sedated MRI in infants and young children. In: Proceedings of New Horizons in $\mathrm{Hu}$ man Brain Imaging, Oahu, Hawaii. March 5-7, 2014 